\title{
Different effects of eyelid blinks and target blanking on saccadic suppression of displacement
}

\author{
HEINER DEUBEL \\ Ludwig-Maximilians-Universität, Munich, Germany \\ BRUCE BRIDGEMAN \\ University of California, Santa Cruz, California \\ and \\ WERNER X. SCHNEIDER \\ Ludwig-Maximilians-Universität, Munich, Germany
}

\begin{abstract}
Displacements of visual stimuli during saccadic eye movements are often not noticed. We have demonstrated that saccadic suppression of image displacement can be eliminated by blanking the stimulus for a short period during and after the saccade (Deubel, Schneider, \& Bridgeman, 1996). Here we report an experiment in which target visibility was interrupted after the saccade, either by distal target blanking or by voluntary eyeblink. The data show that the effect of blinking is different from blanking; interruption of vision due to a blink did not enable subjects to detect target displacements any better than they had done in the no-blank condition. The results provide evidence for an extraretinal signal that distinguishes between endogenous and exogenous sources of temporary object disappearance after the saccade.
\end{abstract}

Saccadic suppression is a reduction of the sensitivity to visual events occurring before, during, and immediately after saccadic eye movements. Two separate types of saccadic suppression should be carefully distinguished. The first type was probably first described by Dodge in 1898, who noted that words recognizable in the periphery during reading pauses could not be seen during saccades. The initial rediscovery of saccadic suppression was in a qualitative report by Ditchburn (1955), followed by an independent rediscovery of the effect by Wallach and Lewis (1966). Since then, many studies have investigated the visual sensitivity to short flashes presented around the time of the saccade (for reviews, see Matin, 1974; Ross, Morrone, Goldberg, \& Burr, 2001). Typically, these investigations reported a moderate elevation of visual threshold (two- to threefold) for detecting spots of light flashed briefly during saccades, while others used more complex visual stimuli, such as sine gratings. Their results have demonstrated that saccadic suppression is strongest for low spatial frequencies (below $1 \mathrm{c} / \mathrm{deg}$ ), whereas higher spatial frequencies remain largely unaffected (Burr, Morrone, \& Ross, 1994; Wolf, Hauske, \& Lupp, 1978, 1980). The selectivity of suppression to the magnocellular pathway strongly suggests that this type of suppression is specific to motion signals (Ross et al., 2001).

The study was supported by the Deutsche Forschungsgemeinschaft (De336/2). Correspondence should be addressed to H. Deubel, Department Psychologie, Ludwig-Maximilians-Universität, Leopoldstr. 13, D-80802 Munich, Germany (e-mail: deubel@psy.uni-muenchen.de).
The second type of saccadic suppression concerns the strong reduction in sensitivity (by three to four log units) for detecting the spatial displacement of a single target or of the whole visual array, when this displacement occurs shortly before or during a saccade (Bridgeman, Hendry, \& Stark, 1975). During fixation, the sensitive motion detectors of the visual system allow perfect perception of even very small jumps of visual objects. Due to the high retinal velocity during a saccade, however, these motion signals are basically eliminated with each eye movement. Without direct evidence for a target jump from motion detectors, detection of intrasaccadic image displacement depends on the comparison of the pre- and postsaccadic target locations. The finding that small image displacements are hard to perceive, therefore, seems to imply that the required precise comparison is normally not performed, that transsaccadic memory about the location of objects is not available to the visual system, or that it is very poor.

However, we have recently provided evidence that a precise memory of presaccadic target location is indeed transferred across the saccade, but that this information is normally not used in displacement detection. In these studies, we demonstrated that blanking a target during a saccade, and presenting it again only $50-300 \mathrm{msec}$ after the saccade ends, restores the detectability of even quite small displacements, which we called the blanking effect (Deubel \& Schneider, 1994; Deubel, Bridgeman, \& Schneider, 1998; Deubel, Schneider, \& Bridgeman, 1996). The blanking effect occurs even for targets in darkness, meaning that displacement detection under this condition can rely on extraretinal signals rather than on retinal information from the structured environment. 
Obviously, the considerable accuracy with which subjects can judge transsaccadic displacements in the blanking condition requires both the maintenance of high-quality information about presaccadic target position across the saccade and a precise extraretinal signal. Thus, it follows from our findings that precise information about the presaccadic target position and a precise extraretinal signal are indeed available for stimulus localizations after the saccade, but they ordinarily are not used in perception. We have suggested that this is because the visual system assumes, as a null hypothesis, the stability of any object that is continuously available both before and after the saccade. Only a very large discrepancy between eye movement magnitude and image position can break this assumption. It is also broken when the presaccadic object is not present immediately after the saccade. Only under this condition are precise transsaccadic information and extraretinal signals used to achieve displacement detection.

These previous studies investigated spatial and temporal constraints of the effect of an exogenous blanking of the stimulus (Deubel et al., 1998; Deubel et al., 1996; Deubel, Schneider, \& Bridgeman, 2002). However, there also exists a natural case in which visual information is temporarily unavailable - namely, during an eyeblink. Indeed, many large saccades are accompanied by blinks. Stark (1968), using an analog photoelectric method, showed that blinks can obscure the pupil for about $200 \mathrm{msec}$. Moreover, it has been demonstrated that the onsets of blinks and of (vertical) saccades are very well synchronized (Becker \& Fuchs, 1988). Interestingly, the perceptual consequences of blinks are much smaller than the consequences obtained from a similar interruption of visual input during fixation: The blink is perceived as being much shorter than it really is (Volkmann, Riggs, \& Moore, 1980).

The question arises whether blinks that occur during saccades can induce a blanking effect. A blink extending beyond the end of the saccade induces an endogenous postsaccadic gap in the visual information flow, visually similar to a distal target disappearance. If blinks can function like the blanking intervals demonstrated in our previous research, we would expect subjects to be capable of detecting small jumps of a target during a saccade accompanied by a blink, even if the distal stimulus is continuously present. If the visual system does not process blinks as interruptions of information flow, however, performance should be comparable to that in the no-blank case. Since the retinal information is identical in both conditions, such a finding would imply that nonvisual, extraretinal information signals whether the eyes are open, influencing the processing of the postsaccadic target. To study this question, subjects were instructed to perform a voluntary blink during their saccade. The effect of the blink on displacement detection was compared with the effect of an exogenous blanking of the target.

\section{METHOD}

\section{Subjects}

Four male subjects (ages 26-44) participated in the experiment; one of them was one of the authors of this study (H.D.). The other 3 were also laboratory staff members, but they were naive about the purpose of the experiments. The data of a 5th subject (B.B.) could not be used in the final analysis since the flash detection experiment (see below) revealed that he did not completely close his eyes while blinking.

\section{Apparatus and Stimuli}

The stimulus consisted of a small red laser dot (diameter $0.2^{\circ}$ ), back-projected via a fast $x-y$ mirror galvanometer system on an opaque screen in total darkness. The laser dot could be switched off and on by means of special purpose hardware within $2 \mathrm{msec}$. The mirror galvanometers allowed shifting of the target by $10^{\circ}$ within about $3 \mathrm{msec}$; during the shift, the dot was switched off to prevent a visual streak on the screen. Viewing distance was $1.5 \mathrm{~m}$, and vision was binocular.

Movements of the right eye were recorded with a two-dimensional search coil based on the principle of Robinson (1963). Three sets of magnetic coils mounted orthogonally in a cubic coil frame $(70 \mathrm{~cm}$ frame size along an edge) were driven with high-frequency alternating current $(20 \mathrm{kHz})$ in phase quadrature, inducing currents in a small coil embedded in a toroidal contact lens (Skalar Medical, Delft). The lens was held firmly in place on the saddle-shaped surface at the edge of the limbus of the eye. The induced currents were recorded from two fine wires emanating from the search coil. After amplification and phase-locked detection, two analog signals were obtained which represented the sine of the horizontal and vertical components of the contact lens orientation. The system was insensitive to head translations within the region of uniform field. It provided a noise level less than $1 \mathrm{~min}$ of arc peak to peak and a linearity error of less than $.25 \%$.

The analog signals corresponding to horizontal and vertical eye movement were digitized at a frequency of $500 \mathrm{~Hz}$ and stored on disk for off-line analysis. A two-point central difference differentiation algorithm (Bahill, Kallman, \& Lieberman, 1982) continuously estimated the momentary eye velocity and generated a trigger signal indicating the occurrence of a saccade once the velocity exceeded $15 \% \mathrm{sec}$. We verified that the trigger-induced target blanking or target shift consistently occurred in the first third of the duration of the saccade.

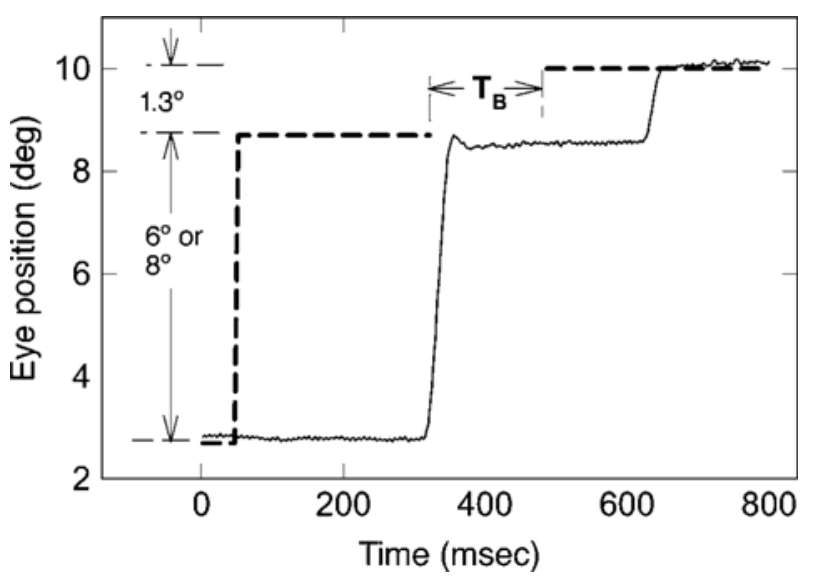

Figure 1. Target position (dashed curve) and eye position (solid curve) in a trial with stimulus blanking. The target jumped left or right by $6^{\circ}$ or $8^{\circ}$ to elicit a saccade. Triggered by the onset of the saccade, the target was blanked and reappeared after the blanking interval $T_{B}$, at a location displaced by $1.3^{\circ}$ with respect to the previous target position. The subject's assignment was to follow the target and to report the direction of the second target displacement. 


\section{Procedure}

Two different experimental blocks ("no blink" and "with blink") were run. Figure 1 displays a typical example of a "no blink" trial in which the solid curve represents the eye movement while the dashed curve shows target shift and blanking. The subject was instructed to follow an initial jump of the laser dot by $6^{\circ}$ or $8^{\circ}$, to the left or to the right.

The onset of the eye movement triggered a saccade-contingent blanking of the target which lasted 0 (no blank), 150, 250, or $400 \mathrm{msec}$ (the case shown in the figure refers to a blanking interval of $150 \mathrm{msec}$ ). The target then reappeared at a position displaced $1.3^{\circ}$ from its original position, either in the same or in the opposite direction of the initial jump. At the end of each trial, the subject's task was to indicate by buttonpress whether the secondary displacement had occurred in the same direction as the initial jump or in the opposite direction. The target remained on until the end of the trial; its final position was the starting position for the next trial. In the "no blink" blocks, the subject was instructed not to blink during the trial. In the "with blink" blocks, the subject was asked to perform a voluntary blink, simultaneous with the saccade. Before data recording began, the subject practiced saccade-contingent blinking with feedback. Each subject performed three blocks of each type, each block containing 96 single trials.

In the critical experimental condition including the blinks, the recorded eye movement traces were used to analyze the occurrence and temporal properties of the eyeblinks. This was possible due to high spatial accuracy of the eye coil signals, in which the occurrence of a blink could easily be discriminated from a normal saccade without a blink. The curves in Figure 2 give examples of recorded horizontal and vertical eye position's in a trial without blink (left graphs) and a trial including a blink during the saccade (right graphs). It can be seen that the blink typically results in a distinct artifact, which is particularly clear in the vertical channel. The prominent negative deviation of the vertical eye position signal in the blink case indicates a transient downward component of the eyes that is known to accompany eyeblinks (Collewijn, van der Steen, \& Steinman, 1985). Using specialized custom-made software, we analyzed each blink trial manually to mark the endpoint of the deviation that was taken to indicate the end of the blink (see Figure 2). Since it was difficult to discriminate the start of the blinks from saccade onsets in the traces, we did not mark and analyze blink onsets or durations.

An important question was how well these times of the end of the artifact coincided with the onset of vision after each blink. For this purpose, a "flash detection" control experiment was performed in which each trial first started with the laser dot jumping by $6^{\circ}$ or $8^{\circ}$, to the left or to the right. The laser was blanked after $100 \mathrm{msec}$. The subject followed the target jump with a saccade combined with a voluntary, simultaneous blink. Triggered by the saccade, in $71 \%$ of the trials a 5-msec flash of the laser dot was presented at various times during and after the blink, at the target position. In the remaining $29 \%$ of the trials, no flash was presented. The subject finally indicated, by pressing one of two buttons, whether he had detected the test flash. Each subject performed a total of 280 of these trials.

\section{RESULTS}

The results of the "flash detection" control experiment are given in Figure 3, showing the rate of flash detection as a function of the interval between the end of the blink
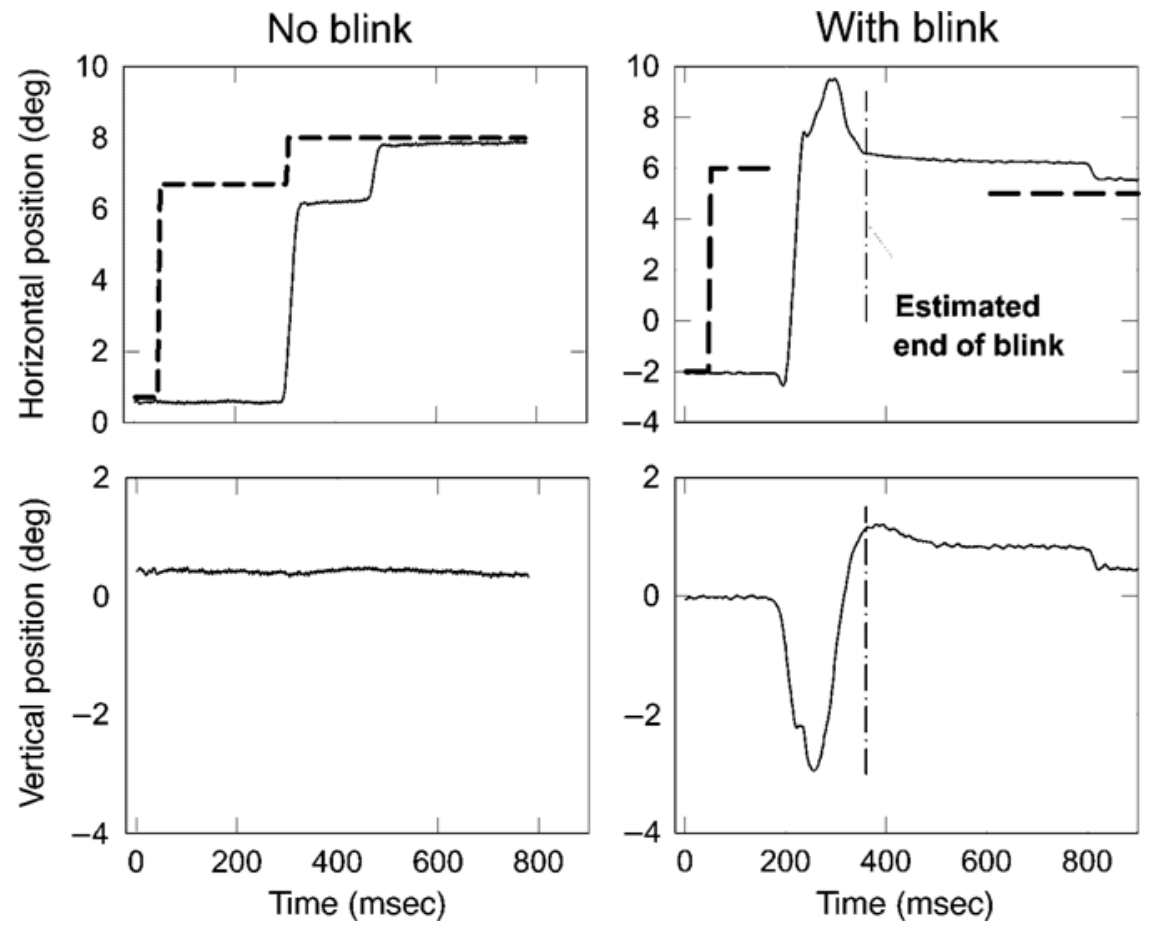

Figure 2. Examples for eye movement traces recorded with the search coil technique in a trial without eyeblink (left plots) and in a trial in which the saccade was accompanied by a voluntary eyeblink (right plots). The dashed curves indicate the horizontal target position. Solid curves, upper panels: Horizontal eye position. Solid curves, lower panels: Vertical eye position. The traces show that the blink leads to a distinct artifact both in the horizontal and in the vertical eye position signals. In order to estimate the time of blink end, the trials were analyzed manually to mark the endpoints of the blink artifacts (vertical dashed-dotted lines). 


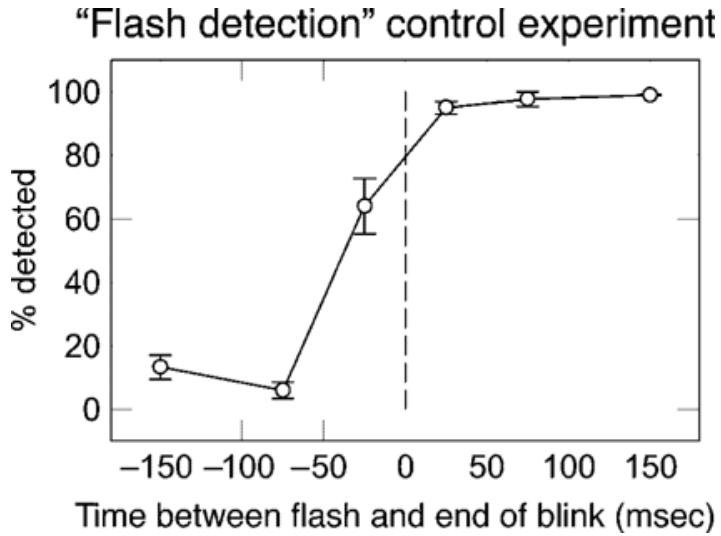

Figure 3. Control experiment in which the target was flashed for $5 \mathrm{msec}$, during or after the blink. Data show percentage probability for the detection of the flash as a function of the time between flash presentation and the end of the blink artifact (see Figure 2).

artifact (see Figure 2) and flash occurrence. The vertical bars indicate standard errors. Obviously, the end of the blink-induced artifact in the eye movement traces correlated very well with the onset of vision after the blink. The time at which the subjects reached $50 \%$ flash detection ranged from 28 to $38 \mathrm{msec}$ before the end of the artifact, yielding a mean of $34 \mathrm{msec}$. The false alarm rate was zero. It is very unlikely that the visibility of the stimulus was affected by saccadic suppression or by blink suppression, since the brightness of the laser spot was very high. The "flash detection" control experiment thus demonstrates that the times marked in the blink analyses can be used as a reliable measure for the onset of visual stimulation after blinks. In order to account for the finding that vision started, on the average, $34 \mathrm{msec}$ before the end of the artifact, we corrected in the following the end of each blink artifact by this value, finally obtaining an estimate for the time when visual input became available after the blink in each trial.

Figure 4 shows the percentage of correct discrimination for onward and backward target jumps as a function of the blanking period, for each of the 4 subjects. The data from the "no blink" blocks are presented as open circles, and those from the "with blink" blocks are presented as filled circles. The solid vertical line indicates the approximate end of the primary saccade, and the dashed vertical line indicates the mean onset time for vision after the blink for each subject, resulting from the analysis of the blink artifact described above.

It can be seen that the data of the "no blink" trials reproduce the blanking effect: Discrimination performance is close to chance for zero blanking but rises steeply for longer blank durations. For the blocks that include a blink, performance also depends on target blanking. However, now performance improves only with considerably longer blanking durations: The curves are shifted by about $150 \mathrm{msec}$ to longer blanking durations, which roughly corresponds to the postsaccadic duration of the blink. A two-way analysis of variance on the percentage of correct responses, with subjects as replications, showed highly significant effects of the blanking interval $\left[F(3,9)=39.9, M S_{\mathrm{e}}=28.3\right.$, $p<.001]$ and of blinking $\left[F(1,3)=35.5, M S_{\mathrm{e}}=12.2\right.$, $p=.009]$. The interaction of both variables also reached significance $\left[F(3,9)=12.7, M S_{\mathrm{e}}=17.7, p=.001\right]$. Somewhat surprisingly, performance with zero blanking interval was slightly better in the "no blink" than in the "with blink" condition $[t(3)=4.7 ; p=.02]$. The transients induced by the blink possibly bias the visual system, to some degree, to accept location changes in the visual world across saccades.

Two important implications follow from this data pattern. First, the endogenously induced absence of a stimulus after a saccade due to a blink is interpreted differently from the absence due to a distal target blanking. This can best be illustrated by the difference in performance at a blanking duration of $150 \mathrm{msec}$ which is close to the beginning of viewing at the end of the blink. For both the "no blink" and "with blink" conditions, vision of the target becomes available at about the same time. Nevertheless, performance for the exogenous target blanking is far superior to that for the endogenous, blink-induced blank. Thus, the interruption of vision due to the blink did not enable the subjects to do any better than they had done in the "no blink" condition. Second, even after the end of a blink, subsequent blanking of the target allows for the detection of its displacement. For all 4 subjects, performance improves monotonically with longer postblink blanking intervals.

\section{DISCUSSION}

The experiment compared the effects of endogenous eyeblinks and exogenous target blanking on saccadic suppression of displacement. As can be seen from the "flash detection" control experiment, the interruption of vision during a blink is just as complete, and just as long-lasting, as the interruption during many of our blanking experiments (Deubel et al., 1996). Despite this interruption, however, blinks do not elicit a blanking effect; they do not facilitate detection of differences between presaccadic and postsaccadic target positions. This implies that the space constancy system is informed that an interruption of vision is due to a blink and does not start to compute postsaccadic target location during the blink if the saccade and the blink occur simultaneously.

What possible sources of information would allow the visual system to distinguish between exogenous and blinkinduced target disappearance? In the natural world, the blink is characterized by a uniform field of low brightness, while an obscured saccade target can be a relatively small feature in a large field of objects and textures. In the laboratory situation of our experiment, however, the retinal consequences of eyeblinks and target blanks are largely identical. A blink is extremely rapid, reaching eyelid velocities of up to $2,000^{\circ} / \mathrm{sec}$ during its down-phase and up to $700^{\circ}$ /sec during its up-phase (Evinger, Manning, \& Si- 
saccade end viewing onset
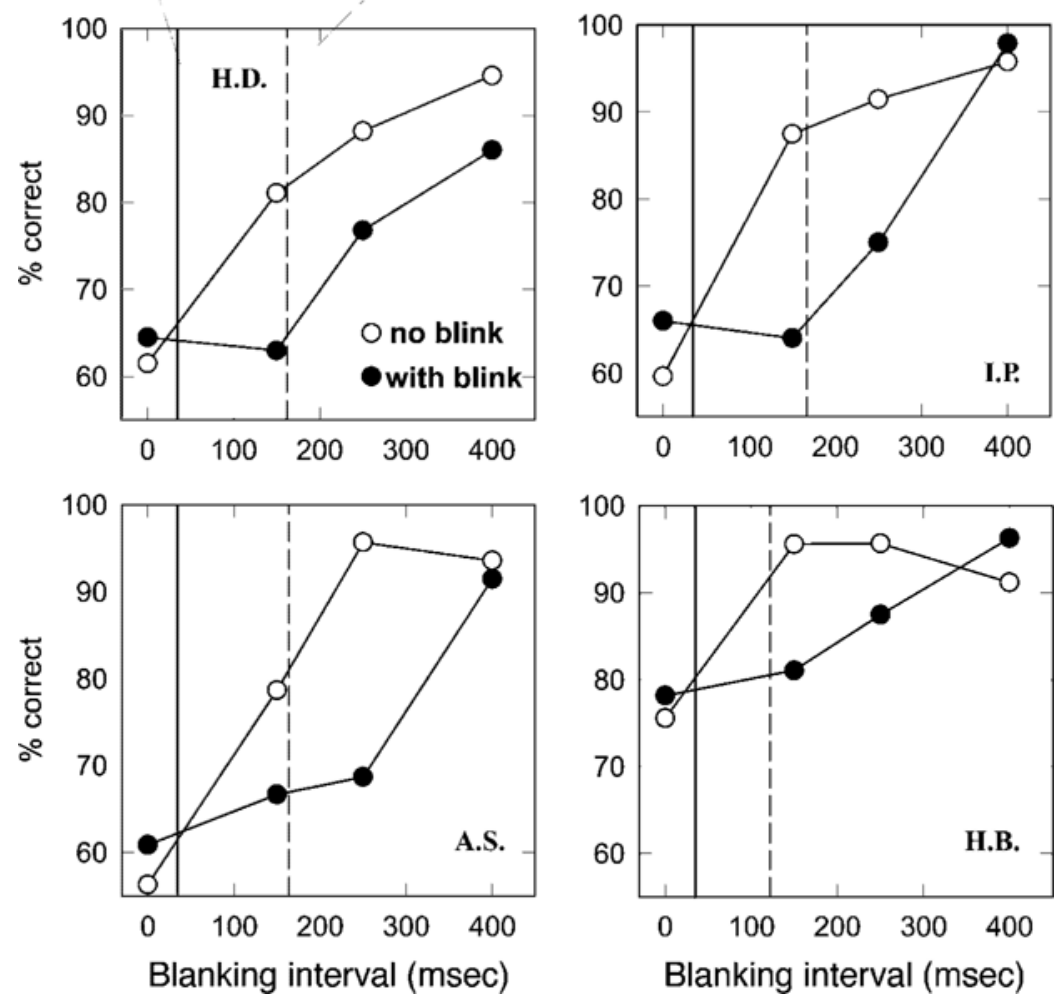

Figure 4. Discriminability of direction of target displacement as a function of blanking interval $T_{B}$, for the 4 subjects. The open circles show the discrimination performance from trials without eyeblinks. The filled circles show the data following a combined saccade and blink, plus a varying blanking interval. The solid vertical line in each plot indicates the approximate end of the saccade, and the dashed vertical line displays the mean estimated onset time of vision after the blink for each subject.

bony, 1991). The time it takes the lid to transit the central region of the pupil is primarily important for the detailed time course of the visual transient. Given the high eyelid velocities during a voluntary blink, this time can be estimated to within a few milliseconds. In other words, the target disappears and reappears abruptly, with no other change in retinal stimulation. Therefore, we conclude from our findings that the extraretinal information about the status of the eyeblink system must be used to distinguish between the visual effects of blinking and blanking.

Every time we blink our eyes, the image of the retina goes almost completely dark. Nevertheless, we hardly notice these interruptions, even though a similar external darkening is startling (Volkmann, Riggs, \& Moore, 1980). Volkmann et al. first demonstrated that suppression of flashed visual stimuli occurs not only with saccadic eye movements but also with eyeblinks. By shining a light through the roof of the mouth of human subjects to permit retinal illumination independent of eyelid position, these authors showed that there is a reduced sensitivity to light around the time of blink onset, a phenomenon known as "blink suppression." In the following, a number of inves- tigators have shown that the suppression of sensitivity around blinks has similar psychophysical properties to the suppression seen in saccadic eye movements, suggesting that the same mechanism might be involved in both cases (see, e.g., Ridder \& Tomlinson, 1993, 1995, 1997; Stevenson, Volkmann, Kelly, \& Riggs, 1986).

Obviously, different kinds of transients have different perceptual impacts: The sudden change of the retinal image of a scene due to a saccade or a blink does not disrupt the perceptual stability and continuity as much as a similar exogenous change would. Therefore, in at least some respects the visual system must process various kinds of transient events differently. Recently, Gawne and Martin $(2000,2002)$ studied the activity of neurons in various areas of the visual cortex under four different conditions: (1) the stimulus flashing on and off while the eye was fixating; (2) external darkening of the entire scene; (3) stimulus onset and offset induced by a saccade; and (4) offset induced by an eyeblink. The data show that most neurons responded to the onsets and offsets in a very similar manner, independently of how the blanking was induced. However, in a substantial minority of cells the response varied 
strongly as a function of the transient event. So some neurons in V1 responded with a transient burst of activity to the onset of an external darkening but not to a blink, suggesting that the suppression of this transient causes us to ignore blinks.

The fact that a blink does not act as a blank is probably useful in normal visual perception. Several authors (see, e.g., Bridgeman, van der Heijden, \& Velichkovsky, 1994) have hypothesized that saccadic suppression of image displacement plays an important role in preserving perceived stability across saccades; it serves to bridge the errors between extraretinal sources of location information and the actual movement. Deubel et al. (1996) have shown that saccadic suppression of image displacement disappears with a postsaccadic target blanking. Therefore, if the visual system would treat postsaccadic target absence due to a blink just like a distal target blanking, a saccade accompanied by a blink would often result in perceived instability of the visual world.

In our previous studies, we demonstrated that saccadic suppression of image displacement largely disappears when postsaccadic target information is absent immediately after the saccade (Deubel et al., 1996) and that stimuli present after the saccade are taken as a spatial reference for localization (Deubel et al., 1998; Deubel et al., 2002). This resulted in a new interpretation of the mechanism of transsaccadic space constancy. The finding presented here further specifies properties of the blanking effect and has some theoretical implication as to how visual stability is preserved across saccadic eye movements. According to our theoretical interpretation, visual attention shifts to the saccade target before the saccade is executed (for empirical evidence, see Deubel \& Schneider, 1996; Hoffman \& Subramaniam, 1995). Due to the attention shift, location and visual attributes of the target and of surrounding objects are stored in transsaccadic memory. Presumably, the capacity of transsaccadic memory is limited to a few, possibly not more than four, visual objects (Irwin, 1992, 1993). After the saccade, the visual system searches for the previous object of attention within a restricted spatiotemporal "constancy window" (McConkie \& Currie, 1996). The constancy window is about $50 \mathrm{msec}$ in duration, and it is confined to a limited spatial area of a few degrees around the saccade target (Deubel, 2004). If the object is found, the visual world is assumed to be stable. Spatial information from the previous fixation is discarded or ignored, and localization proceeds using the currently available information. The results from the blink experiment suggest that, in the case of eyeblinks, extraretinal information about the eyeblink status is used to inform the space constancy system that the object should not be sought until the eyes are open.

When, as due to a distal target blanking, the target is not found, the assumption of stability of the visual world is broken. Extraretinal signals are used to establish the new target location. Only in this case does the system use stored information about sensory conditions such as tar- get position before the saccade, which results in the detection of intrasaccadic displacements.

\section{REFERENCES}

Bahill, A. T., Kallman, J. S., \& Lieberman, J. E. (1982). Frequency limitations of the two-point central difference differentiation algorithm. Biological Cybernetics, 45, 1-4.

BECKER, W., \& FuCHS, A. F. (1988). Lid-eye coordination during vertical gaze changes in man and monkey. Journal of Neurophysiology, $\underline{\mathbf{6 0}}_{2}$ 1227-1252.

Bridgeman, B., Hendry, D., \& STARK, L. (1975). Failure to detect displacement of the visual world during saccadic eye movements. Vision Research, 15, 719-722.

BRIDGEMAN, B., vAN DER HeIJden, A. H. C., \& VelichKovsky, B. M. (1994). A theory of visual stability across saccadic eye movements. Behavioral \& Brain Sciences, 17, 247-292.

Burr, D. C., Morrone, M. C., \& Ross, J. (1994). Selective suppression of the magnocellular visual pathway during saccadic eye movements. Nature, 371, 511-513.

Collewisn, H., van Der Steen, J., \& Steinman, R. M. (1985). Human eye movements associated with blinks and prolonged eyelid closure. Journal of Neurophysiology, 54, 11-27.

DEUBEL, H. (2004). Localization of targets across saccades: Role of landmark objects. Visual Cognition, 11, 173-202.

Deubel, H., Bridgeman, B., \& SCHN EIDER, W. X. (1998). Immediate post-saccadic information mediates space constancy. Vision Research, 38, 3147-3159.

DeUbel, H., \& Schneider, W. X. (1994). Can man bridge a gap? Behavioral \& Brain Sciences, 17, 259-260.

Deubel, H., \& Schneider, W. X. (1996). Saccade target selection and object recognition: Evidence for a common attentional mechanism. Vision Research, 36, 1827-1837.

Deubel, H., SchneIDER, W. X., \& Bridgeman, B. (1996). Postsaccadic target blanking prevents saccadic suppression of image displacement. Vision Research, 36, 985-996.

Deubel, H., SchneIDer, W. X., \& Bridgeman, B. (2002). Transsaccadic memory of position and form. In J. Hyönä, D. Munoz, W. Heide, \& R. Radach (Eds.), The brain's eye: Neurobiological and clinical aspects of oculomotor research (pp. 165-180). Amsterdam: Elsevier.

DitchbuRN, R. W. (1955). Eye movements in relation to retinal action. Optic Acta, 1, 171-176.

Evinger, C., Manning, K. A., \& Sibony, P. A. (1991). Eyelid movements - mechanisms and normal data. Investigative Ophthalmology \& Visual Science, 32, 387-400.

GAWNE, T. J., \& MARTIN, J. M. (2000). Activity of primate V1 cortical neurons during blinks. Journal of Neurophysiology, 84, 2691-2694.

Gawne, T. J., \& MARTIN, J. M. (2002). Responses of primate visual cortical neurons to stimuli presented by flash, saccade, blink, and external darkening. Journal of Neurophysiology, 88, 2178-2186.

Hoffman, J. E., \& Subramaniam, B. (1995). The role of visual attention in saccadic eye movements. Perception \& Psychophysics, $\underline{\mathbf{5 7}}_{2}$ 787-795.

IRWIN, D. E. (1992). Memory for position and identity across eye movements. Journal of Experimental Psychology: Learning, Memory, \& Cognition, 18, 307-317.

IRWIN, D. E. (1993). Memory for spatial position across saccadic eye movements. In G. d'Ydewalle \& J. van Rensbergen (Eds.), Perception and cognition: Advances in eye movement research (pp. 323-332). Amsterdam: North-Holland.

Matin, E. (1974). Saccadic suppression: A review and an analysis. Psychological Bulletin, 81, 899-917.

MCCONKIE, G. W., \& CURRIE, C. B. (1996). Visual stability across saccades while viewing complex pictures. Journal of Experimental Psychology: Human Perception \& Performance, 22, 563-581.

RiDDER, W. H., \& ToMLINSON, A. (1993). Suppression of contrast sensitivity during eyelid blinks. Vision Research, 33, 1795-1802.

RidDER, W. H., \& TomLinson, A. (1995). Spectral characteristics of blink suppression in normal observers. Vision Research, 35, 2569-2578. 
RidDER, W. H., \& TomLinson, A. (1997). A comparison of saccadic and blink suppression in normal observers. Vision Research, 37, 3171-3179. Robinson, D. A. (1963). A method for measuring eye movements using a scleral search coil in a magnetic field. IEEE Transactions in Biomedical Engineering, 26, 137-145.

Ross, J., Morrone, C. M., GoldBerG, M. E., \& Burr, D. C. (2001). Changes in visual perception at the time of saccades. Trends in Neurosciences, 24, 113-121.

StARK, L. (1968). Neurological control systems. New York: Plenum. Stevenson, S. B., Volkmann, F. C., Kelly, J. P., \& Riggs, L. A. (1986). Dependence of visual suppression on the amplitudes of saccades and blinks. Vision Research, 26, 1815-1824.

Volkmann, F. C., RigGS, L. A., \& Moore, R. K. (1980). Eyeblinks and visual suppression. Science, 207, 900-902.
Wallach, H., \& LewIS, C. (1966). The effect of abnormal displacement of the retinal image during eye movements. Perception \& Psychophysics, 1, 25-29.

Wolf, W., Hauske, G., \& Lupp, U. (1978). How presaccadic gratings modify postsaccadic modulation transfer function. Vision Research, 18, 1173-1179.

WOLF, W., HAUSKE, G., \& LUPP, U. (1980). Interaction of pre- and postsaccadic patterns having the same coordinates in space. Vision Research, 20, 117-124.

(Manuscript received March 10, 2003; revision accepted for publication October 7,2003.) 